\section{MS27-03 Structure refinement using 'digital' electron diffraction}

Richard Beanland ${ }^{1}$

1. University of Warwick

email: r.beanland@warwick.ac.uk

Electron diffraction datasets that cover a large angular range can now be constructed from hundreds or thousands of convergent beam electron diffraction patterns using computer control of a transmission electron microscope. These 'digital' large angle convergent beam electron diffraction (D-LACBED) patterns contain a wealth of data that can in principle be used to refine structures and electron densities to unprecedented accuracy. We explore refinement strategies that match simulated D-LACBED patterns to experimental data, using the changing structure of $\mathrm{Ca} 3 \mathrm{Mn} 2 \mathrm{O} 7$. This Ruddlesden-Popper compound exhibits a tilt of the oxygen octahedra about the long axis of the unit cell that appears to vary with temperature, as well as a second octahedral tilt, about an axis perpendicular to the first, that switches abruptly at a first order phase transformation around 350C.In order to obtain structural or electron density models that match experimental results, we have developed an open source Bloch-wave program, Felix. This software platform has been written clearly and concisely with a strong emphasis on architecture to aid further development. We show refinements based on atomic location and Debye-Waller factors and consider different approaches to find the best global fit to the data.

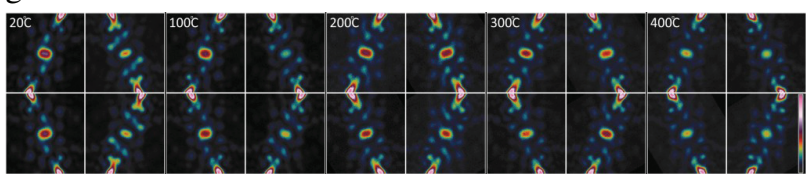

Figure 1. Sets of four 422-type D-LACBED patterns. At room temperature a horizontal mirror is present but no vertical one, indicating a spacegroup of $\mathrm{Cmc} 21$. The patterns become more symmetrical up to $300 \mathrm{C}$, indicating $\mathrm{Cmcm}$. Finally, intensities change abruptly when the material transforms to I4/mmm (400C).

Keywords: Structure refinement, Bloch wave, digital electron diffraction, $\mathrm{Ca} 3 \mathrm{Mn} 2 \mathrm{O} 7$

\section{MS27-O4 Fast electron diffraction tomography}

Mauro Gemmi ${ }^{1}$, Mari Grazia Immacolata La Placa ${ }^{1}$, Athanassios Galanis $^{2}$, Edgar F. Rauch ${ }^{3}$, Stavros Nicolopoulos ${ }^{2}$

1. Ceneter for Nanotechnology Innovation@NEST, Istituto Italiano di Tecnologia, Pisa, Italy

2. NanoMEGAS SPRL, Brussels, Belgium

3. SIMAP CNRS-Grenoble INP, Saint Martin d'Hères, France

email: mauro.gemmi@iit.it

Electron diffraction tomography (EDT) has completely changed the possibility of solving crystal structures using electron diffraction data. Electron diffraction tomography data, if collected in precession mode[1] or in rotation mode[2] are close enough to a kinematical approximation to be successfully used in ab-initio structure solution methods designed for kinematical scattering, like direct methods or charge flipping. In an EDT data collection the patterns are recorded while the crystal is tilted around the goniometric axis, therefore the crystal must be kept under the electron beam during rotation. The availability of a procedure for collecting automatic EDT on a nanocrystal without any recentring procedure would speed up the data collection, increase the number of crystals which could be analyzed for each TEM session, and reduce the electron dose suffered by the crystal. Thanks to the goniometer stability of a Zeiss Libra 120 electron microscope, we have developed an automatic data collection procedure which allows a $40^{\circ}$ range data collection without any intervention of the operator in a total time of 2 minutes. Such a small angular range is suitable for unit cell determination and can be used as a phase screening method to identify new unknown phases on a multiphase sample. In some special cases, depending on the position in the sample grid, the dimension of the crystal and the sample holder type, the automatic procedure can collect a larger angular range, between $60^{\circ}$ and $80^{\circ}$, suitable for structure solution. By playing on the tilt speed, the exposure time and the detector speed (increasing the readout by binning) the procedure can be adapted to collect precession assisted EDT (PEDT), rotation EDT (REDT) or a different EDT type, that we have called integrated EDT (IEDT), in which the reciprocal space between the patterns is sampled during the exposure, like what happens in $\mathrm{x}$-ray single crystal diffraction with area detector. The method has been tested on a known crystal structure, $\mathrm{MgMoO}_{4}$, which has been solved with data collected in the three data collection modes, PEDT, REDT and IEDT.

\section{References.}

[1] Mugnaioli, E., Gorelik, T. E. \& Kolb, U. (2009). Ultramicroscopy, 109, 758-765.

[2] Zhang D., Oleynikov P., Hovmöller S., Zou X. D. (2010). Z. Kristallogr. 22594.

Keywords: Electron diffraction tomography, precession electron diffraction 